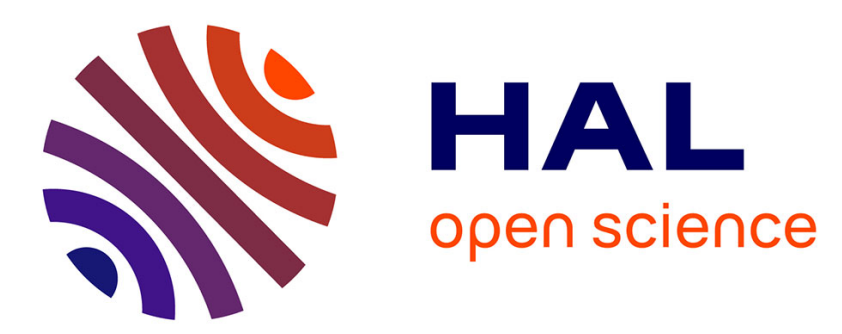

\title{
Global analysis of a schistosomiasis infection model with biological control
}

\author{
Mouhamadou Diaby, Abderrahman Iggidr, Mamadou Sy, Abdou Sene
}

\section{To cite this version:}

Mouhamadou Diaby, Abderrahman Iggidr, Mamadou Sy, Abdou Sene. Global analysis of a schistosomiasis infection model with biological control. Applied Mathematics and Computation, 2014, 246, pp.731 - 742. 10.1016/j.amc.2014.08.061 . hal-01086737

\section{HAL Id: hal-01086737 https://hal.inria.fr/hal-01086737}

Submitted on 24 Nov 2014

HAL is a multi-disciplinary open access archive for the deposit and dissemination of scientific research documents, whether they are published or not. The documents may come from teaching and research institutions in France or abroad, or from public or private research centers.
L'archive ouverte pluridisciplinaire HAL, est destinée au dépôt et à la diffusion de documents scientifiques de niveau recherche, publiés ou non, émanant des établissements d'enseignement et de recherche français ou étrangers, des laboratoires publics ou privés. 


\title{
Global analysis of a schistosomiasis infection model with biological control
}

\author{
Mouhamadou Diaby ${ }^{\mathrm{a}, \mathrm{b}}$, Abderrahman Iggidr ${ }^{\mathrm{a}}$, Mamadou Sy $^{\mathrm{b}}$, Abdou Sène $^{\mathrm{b}}$ \\ ${ }^{a}$ Inria and Université de Lorraine and CNRS \\ Epi MASAIE, Institut Elie Cartan de Lorraine UMR 7502, ISGMP Bat. A, Metz, \\ F-57045, France. \\ ${ }^{b}$ Laboratoire d'Analyse Numérique et Informatique(LANI), Université Gaston Berger de \\ Saint-Louis, UFR SAT BP 234 Saint-Louis, Sénégal.
}

\begin{abstract}
In this paper, the global stability of a schistosomiasis infection model that involves human and intermediate snail hosts as well as an additional mammalian host and a competitor snail species is studied by constructing Lyapunov functions and using properties of $K$ monotone systems.

We derive the basic reproduction number $\mathcal{R}_{0}$ for the deterministic model, and establish that the global dynamics are completely determined by the values of $\mathcal{R}_{0}$. We show that the disease can be eradicated when $\mathcal{R}_{0} \leq 1$. In the case where $\mathcal{R}_{0}>1$, we prove the existence, uniqueness and global asymptotic stability of an endemic steady state. This mathematical analysis of the model gives insight about the epidemiological consequences of the introduction of a competitor resistant snail species.
\end{abstract}

Keywords: Epidemic models, Nonlinear dynamical systems, Monotone systems, Global stability, Reproduction number, Schistosomiasis.

\section{Introduction}

Schistosomiasis also known as bilharzia is a parasite-induced disease. The prevalence of Schistosomiasis is high in tropical and sub-tropical countries, especially in poor rural regions without access to safe drinking water and

Email addresses: diabloss84@yahoo.fr (Mouhamadou Diaby), abderrahmann.iggidr@inria.fr (Abderrahman Iggidr), syndioum@yahoo.fr (Mamadou Sy), asene1001@yahoo.fr (Abdou Sène) 
adequate sanitation. The parasites, schistosomes, have to go through an intermediate host (snails in most cases) to complete their life cycle: from eggs, to miracidia, to cercaria, finally to adult worms. Schistosomes have two stages of reproduction: the first one consists in a sexual reproduction in the blood vessels of humans and the second stage is an asexual amplification in snails.

Many strategies are used to prevent and control the schistosomiasis infection: chemotherapy, chemical molluscicides, improvement of sanitary conditions, snail control.

For many endemic situations, mass drug administration with praziquantel is recommended by the WHO (World Health Organization)[23]. However, as reported by WHO, "a major limitation to schistosomiasis control has been access to praziquantel. Available data show that only $10 \%$ of people requiring treatment were reached in 2011". The price of a chemical antischistosomial is still too expensive for people in many developing countries.

Biological control by introducing competitive snails of the intermediate snail hosts is a control strategy that may require relatively little funding. For instance, Schistosomiasis infection has declined in Puerto Rico due to the introduction of Thiara granifera, a snail which competes with the intermediate host snail of schistosomes Biomphalaria glabrata (see [17], [1]).

Mathematical modeling and analysis of schistosomiasis has drawn many attentions since the first paper by MacDonald in [15]. Thereafter many others researchers built excellent models and developed a decent understanding of transmission mechanism of schistosomiasis (see $[7,6,24]$ ).

Recently, a schistosomiasis infection model described by E.J Allen and H.D Victory [1] has been proposed. This model generalizes in some way, previous mathematical models such as those described by Anderson and May [2]; Kimbir [13].

Here, we consider the model proposed in [1]. This model allows competition between the intermediate host snails and a resistant snail species in order to study the advantages of biological control. In [1], computational simulations have been done to estimate some parameters and to study control of the infection by chemotherapy and biological control using snail competition.

In this paper, we propose a complete mathematical analysis of the deterministic model of [1]. A stability analysis is provided to study the epidemiological consequences of control strategies. Therefore, the specific objectives are to determine the threshold parameter that measure initial disease transmission and to analyze the steady states stability. This reproduction number can be 
used to assess the potential impact of biological control in the schistosomiasis disease eradication. We show that the DFE is globally asymptotically stable by constructing a Lyapunov function. The existence and uniqueness of a nontrivial equilibrium (endemic equilibrium) is shown by applying a fixed point Theorem due to Hethcote and Thieme [19] and its local asymptotic stability is investigated using a Krasnoselskii sublinearity trick. The global asymptotic stability of this unique endemic equilibrium is shown using properties of K-monotone systems [11].

The paper is organized as follows. In Section 2 we present the model described by E.J Allen and H.D Victory [1]. Its well-posedness is established and a reduced model is proposed. In Section 3, we determine $\mathcal{R}_{0}$ the basic reproductive number of the model and we establish the global asymptotic stability of the disease-free equilibrium when the basic reproductive number is less or equal to one. Section 4 is devoted to the study of the system behavior when the basic reproductive number is larger than one. In this case, by using some properties of K-monotone systems (see [18]), we prove the existence of a unique endemic equilibrium and we show its global asymptotic stability.

Finally, in Section 5 , we present some discussions about the characteristics of the competitor resistant snail species that can be used to eliminate the disease.

\section{Model framework}

We consider the model presented in [1]. In their work, four definite mammalian host sub-populations, three intermediate snail host sub-populations, and a population of resistant competitor snails are considered. We assume that the human births and deaths dynamics can be neglected compared to the infection dynamics.

Further, it is assumed that infected snails and infected mammals do not recover from schistosomiasis as their life span are short in comparison to that for humans. The state variables of the model are:

- $H_{s}(t)$ the susceptible (uninfected) human population size.

- $H_{i}(t)$ the infected human population size.

- $S_{s}(t)$ the susceptible snail host population size. 
- $S_{e}(t)$ the population size of the infected snails which are not yet shedding cercariae (latent population size).

- $S_{i}(t)$ the infected and shedding snail population size(shedding population size) .

- $S_{r c}(t)$ the competitor snail population size(resistant to infection).

- $M_{s}(t)$ the susceptible mammal population size.

- $M_{i}(t)$ the infected mammal population size.

In addition, the population of snails as well as mammals are assumed to be competitive. Birth and death rates for the various sub-populations will be denoted by $b_{i}$ and $d_{i}$. The transmission parameters for the model are:

- $t_{15}=t_{H_{s} S_{i}}=$ transmission rate from infected snails to uninfected humans,

- $t_{32}=t_{S_{s} H_{i}}=$ transmission rate from infected humans to uninfected snails,

- $t_{38}=t_{S_{s} M_{i}}=$ transmission rate from infected mammals to susceptible snail,

- $t_{75}=t_{M_{s} S_{i}}=$ transmission rate from infected snails to susceptible mammals.

Competition parameters are defined for the populations as follows:

$c_{33}$ is the competition parameter between $S_{s}$ and $S_{s}, S_{e}, S_{i}$,

$c_{44}$ and $c_{55}$ are the competition parameters between $S_{e}$ and $S_{i}$, respectively, and $S_{s}, S_{e}$, and $S_{i}$,

$c_{36}$ is the competition parameter for snails $S_{r c}$ with snails $S_{s}, c_{46}$ and $c_{56}$ are defined analogously,

$c_{64}$ is the competition parameter for snails $S_{s}, S_{e}$ and $S_{i}$ with $S_{r c}$,

$c_{66}$ is the competition parameter for $S_{r c}$ with $S_{r c}$,

$c_{77}$ and $c_{88}$ are the competition parameter for the mammals populations.

The recovery rate for infected humans is denoted by $r_{12}$ and $r_{54}$ denotes the rate that the latent snail population $S_{e}$ becomes shedding $S_{i}$. 
The time evolution of the different populations is governed by the following system of equations:

$$
\left\{\begin{aligned}
\frac{d H_{s}}{d t}= & -t_{15} S_{i} H_{s}+r_{12} H_{i} \\
\frac{d H_{i}}{d t}= & t_{15} S_{i} H_{s}-r_{12} H_{i} \\
\frac{d S_{s}}{d t}= & b_{3}\left(S_{s}+S_{e}+S_{i}\right)-t_{32} H_{i} S_{s}-d_{3} S_{s}-c_{33} S_{s}\left(S_{s}+S_{e}+S_{i}\right) \\
& -c_{36} S_{s} S_{r c}-t_{38} S_{s} M_{i} \\
\frac{d S_{e}}{d t}= & t_{32} H_{i} S_{s}+t_{38} S_{s} M_{i}-d_{4} S_{e}-c_{44} S_{e}\left(S_{s}+S_{e}+S_{i}\right) \\
& -c_{46} S_{e} S_{r c}-r_{54} S_{e}, \\
\frac{d S_{i}}{d t}= & r_{54} S_{e}-d_{5} S_{i}-c_{55} S_{i}\left(S_{s}+S_{e}+S_{i}\right)-c_{56} S_{i} S_{r c} \\
\frac{d S_{r c}}{d t}= & b_{6} S_{r c}-c_{64} S_{r c}\left(S_{s}+S_{e}+S_{i}\right)-c_{66} S_{r c} S_{r c}-d_{6} S_{r c} \\
\frac{d M_{s}}{d t}= & b_{7}\left(M_{s}+M_{i}\right)-t_{75} S_{i} M_{s}-c_{77} M_{s}\left(M_{s}+M_{i}\right)-d_{7} M_{s} \\
\frac{d M_{i}}{d t}= & t_{75} S_{i} M_{s}-d_{8} M_{i}-c_{88} M_{i}\left(M_{s}+M_{i}\right) .
\end{aligned}\right.
$$

It is assumed for simplicity that $d_{3}=d_{4}=d_{5}, d_{7}=d_{8}, c_{33}=c_{44}=c_{55}$, $c_{77}=c_{88}$ and $c_{46}=c_{56}=c_{36}$. The total human population $N_{H}=H_{s}+H_{i}$ is constant since $\frac{d N_{H}}{d t}=0$. The total no resistant snails population is denoted $N_{S i}=S_{s}+S_{e}+S_{i}$. Its time-evolution is governed by

$$
\frac{d N_{S i}}{d t}=\left(b_{3}-d_{3}\right) N_{S i}-c_{33} N_{S i}^{2}-c_{36} S_{r c} N_{S i}
$$

It follows that

$$
\frac{d N_{S i}}{d t} \leq\left(b_{3}-d_{3}\right) N_{S i}-c_{33} N_{S i}^{2}=\left(\frac{b_{3}-d_{3}}{c_{33}}-N_{S i}\right) c_{33} N_{S i}
$$

The dynamics of the resistant snails population and the total mammals pop- 
ulation $\left(N_{M}=M_{s}+M_{i}\right)$ are respectively governed by

$$
\begin{aligned}
& \frac{d S_{r c}}{d t}=\left(b_{6}-d_{6}\right) S_{r c}-c_{66} S_{r c}^{2}-c_{64} S_{r c}\left(S_{s}+S_{e}+S_{i}\right) \leq\left(\frac{b_{6}-d_{6}}{c_{66}}-S_{r c}\right) c_{66} S_{r c}, \\
& \frac{d N_{M}}{d t}=\left(b_{7}-d_{7}\right) N_{M}-c_{77} N_{M}^{2}=\left(\frac{b_{7}-d_{7}}{c_{77}}-N_{M}\right) c_{77} N_{M} .
\end{aligned}
$$

Thus the feasible region for the system (1) is

$$
\begin{aligned}
& \mathcal{D}=\left\{\left(H_{s}, H_{i}, S_{s}, S_{e}, S_{i}, S_{r c}, M_{s}, M_{i}\right)\right. \in \mathbb{R}_{+}^{8}: \\
&\left.N_{S i} \leq \frac{b_{3}-d_{3}}{c_{33}}, N_{M} \leq \frac{b_{7}-d_{7}}{c_{77}}, S_{r c} \leq \frac{b_{6}-d_{6}}{c_{66}}\right\}
\end{aligned}
$$

Proposition 2.1. The compact set $\mathcal{D}$ is positively invariant and attracting under the flow described by (1).

Proof. It is sufficient to consider the system on the faces of $\mathcal{D}$ and to show that for each face, the vector fields associated to the system points into the set $\mathcal{D}$.

If $N_{S i}=0$ then $\frac{d N_{S i}}{d t}=0$.

If $N_{S i}=\frac{b_{3}-d_{3}}{c_{33}}$ then $\frac{d N_{S i}}{d t} \leq 0$.

If $N_{M}=0$ then $\frac{d N_{M}}{d t}=0$.

If $N_{M}=\frac{b_{7}-d_{7}}{c_{77}}$ then $\frac{d N_{M}}{d t} \leq 0$.

If $S_{r c}=0$ then $\frac{d S_{r c}}{d t}=0$.

If $S_{r c}=\frac{b_{6}-d_{6}}{c_{66}}$ then $\frac{d S_{r c}}{d t}=-c_{64} \frac{b_{6}-d_{6}}{c_{66}}\left(S_{s}+S_{e}+S_{i}\right) \leq 0$.

Furthermore, the model (1) is well-posed epidemiologically. Hence, it is sufficient to study the dynamics of the basic model in $\mathcal{D}$.

\subsection{Reduction of the system}

We will reduce the stability analysis of (1), to the study of a smaller and simpler system. The following theorem(see [22] ) will permit us to reduce the stability analysis to a smaller system. 
Theorem 2.1 ([22], Theorem 3.1). Consider the following $C^{1}$ system

$$
\left\{\begin{array}{l}
\dot{x}=f(x) ; \quad x \in \mathbb{R}^{n} \quad y \in \mathbb{R}^{m} \\
\dot{y}=g(x, y) \\
\text { with an equilibrium point }\left(x^{*}, y^{*}\right) \text { i.e. } \\
f\left(x^{*}\right)=0 \text { and } g\left(x^{*}, y^{*}\right)=0 .
\end{array}\right.
$$

If $x^{*}$ is globally asymptotically stable $(G A S)$ in $\mathbb{R}^{n}$ for the system $\dot{x}=f(x)$, and if $y^{*}$ is $G A S$ in $\mathbb{R}^{m}$, for the system $\dot{y}=g\left(x^{*}, y\right)$, then $\left(x^{*}, y^{*}\right)$ is (locally) asymptotically stable for (2).

Moreover, if all the trajectories of (2) are forward bounded, then $\left(x^{*}, y^{*}\right)$ is GAS for (2).

We define the proportions of the no resistant snails by: $s_{j}=\frac{S_{j}}{N_{S i}}$ for $j=$ $s, e, i$, and the proportions of mammals $m_{j}=\frac{M_{j}}{N_{M}}$ for $j=s, i$.

Using $\dot{s}_{j}=\frac{\dot{S}_{j}}{N_{S i}}-\frac{\dot{N}_{S i}}{N_{S i}} s_{j}=\frac{\dot{S}_{j}}{N_{S i}}-\left(a_{3}-c_{33} N_{S i}-c_{36} S_{r c}\right) s_{j}$,

$\dot{m}_{j}=\frac{\dot{M}_{j}}{N_{M}}-\frac{\dot{N}_{M}}{N_{M}} m_{j}=\frac{\dot{M}_{j}}{N_{M}}-\left(b_{7}-d_{7}-c_{77} N_{M}\right) m_{j}$, and the fact that $s_{s}+s_{e}+s_{i}=1, m_{s}+m_{i}=1$, simple computations show that the new variables $H_{i}, s_{e}, s_{i}, m_{i}, N_{S i}, S_{r c}$, and $N_{M}$ satisfy:

$$
\left\{\begin{aligned}
\frac{d H_{i}}{d t} & =t_{15}\left(N_{H}-H_{i}\right) s_{i} N_{S i}-r_{12} H_{i}, \\
\frac{d s_{e}}{d t} & =\left(t_{32} H_{i}+t_{38} N_{M} m_{i}\right)\left(1-s_{e}-s_{i}\right)-\left(b_{3}+r_{54}\right) s_{e}, \\
\frac{d s_{i}}{d t} & =r_{54} s_{e}-b_{3} s_{i}, \\
\frac{d m_{i}}{d t} & =t_{75} N_{S i} s_{i}\left(1-m_{i}\right)-b_{7} m_{i}, \\
\frac{d N_{S i}}{d t} & =\overbrace{\left(b_{3}-d_{3}\right)}^{a_{3}} N_{S i}-c_{33} N_{S i}^{2}-c_{36} S_{r c} N_{S i}=X_{1}\left(N_{S i}, S_{r c}\right), \\
\frac{d S_{r c}}{d t} & =\overbrace{\left(b_{6}-d_{6}\right)}^{a_{6}} S_{r c}-c_{64} S_{r c} N_{S i}-c_{66} S_{r c} S_{r c}=X_{2}\left(N_{S i}, S_{r c}\right), \\
\frac{d N_{M}}{d t} & =\left(b_{7}-d_{7}\right) N_{M}-c_{77} N_{M}^{2} .
\end{aligned}\right.
$$


This system is triangular: the last three equations do not depend on the variables $H_{i}, s_{e}, s_{i}, m_{i}$.

The last equation has two equilibria: the trivial one which is unstable and a positive equilibrium $N_{M}^{*}=\frac{b_{7}-d_{7}}{c_{77}}$ which is GAS.

Let us consider the following subsystem:

$$
\left\{\begin{array}{l}
\frac{d N_{S i}}{d t}=\overbrace{\left(b_{3}-d_{3}\right)}^{a_{3}} N_{S i}-c_{33} N_{S i}^{2}-c_{36} S_{r c} N_{S i}=X_{1}\left(N_{S i}, S_{r c}\right), \\
\frac{d S_{r c}}{d t}=\overbrace{\left(b_{6}-d_{6}\right)}^{a_{6}} S_{r c}-c_{64} S_{r c} N_{S i}-c_{66} S_{r c} S_{r c}=X_{2}\left(N_{S i}, S_{r c}\right),
\end{array}\right.
$$

The equilibria of (4) are:

$(0,0)$ which is unstable: two positive eigenvalues $a_{3}$ and $a_{6}$.

$E_{1}=\left(\frac{a_{3}}{c_{33}}, 0\right)$ with eigenvalues $-a_{3}$ and $a_{6}-\frac{c_{64} a_{3}}{c_{33}}=\frac{c_{33} a_{6}-c_{64} a_{3}}{c_{33}}$.

$E_{2}=\left(0, \frac{a_{6}}{c_{66}}\right)$ with eigenvalues $-a_{6}$ and $a_{3}-\frac{c_{36} a_{6}}{c_{66}}=\frac{c_{66} a_{3}-c_{36} a_{6}}{c_{66}}$.

If $c_{33} a_{6}-c_{64} a_{3}<0$ then $E_{1}$ is LAS. If $c_{66} a_{3}-c_{36} a_{6}<0$ then $E_{2}$ is LAS.

The system (4) has a positive equilibrium $E^{*}$ :

$$
\left\{\begin{array}{l}
N_{S i}^{*}=\frac{c_{36} a_{6}-c_{66} a_{3}}{c_{36} c_{64}-c_{33} c_{66}}=\frac{c_{66} a_{3}-c_{36} a_{6}}{c_{33} c_{66}-c_{36} c_{64}} \\
S_{r c}^{*}=\frac{c_{64} a_{3}-c_{33} a_{6}}{c_{36} c_{64}-c_{33} c_{66}}=\frac{c_{33} a_{6}-c_{64} a_{3}}{c_{33} c_{66}-c_{36} c_{64}}
\end{array}\right.
$$

We remark that

$$
N_{S i}^{*}=\frac{c_{66} a_{3}-c_{36} a_{6}}{c_{33} a_{6}-c_{64} a_{3}} S_{r c}^{*}
$$

The equilibrium $E^{*}=\left(N_{S i}^{*}, S_{r c}^{*}\right)$ exists if and only if

1. 1st case: $c_{33} c_{66}-c_{36} c_{64}>0$. In this case the existence of $E^{*}$ implies that $E^{*}$ is LAS and the other are unstable.

2. 2nd case: $c_{33} c_{66}-c_{36} c_{64}<0$. In this case $E^{*}$ exists if $c_{33} a_{6}-c_{64} a_{3}<0$ and $c_{66} a_{3}-c_{36} a_{6}<0$. In this case $E_{1}$ and $E_{2}$ are LAS but $E^{*}$ is unstable. 
We shall assume that $E_{1}$ and $E_{2}$ are unstable which implies that

$$
c_{33} a_{6}-c_{64} a_{3}>0 \text { and } c_{66} a_{3}-c_{36} a_{6}>0 .
$$

This implies

$$
c_{33} c_{66}-c_{36} c_{64}>0 .
$$

In this case $E^{*}$ is LAS: eigenvalues with negative real part.

Let $V$ a lyapunov function defined as follows :

$$
V=\left(N_{S i}-N_{S i}^{*} \log N_{S i}\right)+d\left(S_{r c}-S_{r c}^{*} \log S_{r c}\right) .
$$

Then, its derivative along the solutions of (4) satisfies :

$$
\dot{V}=\left(N_{S i}-N_{S i}^{*}\right)\left(a_{3}-c_{33} N_{S i}-c_{36} S_{r c}\right)+d\left(S_{r c}-S_{r c}^{*}\right)\left(a_{6}-c_{64} N_{S i}-c_{66} S_{r c}\right) .
$$

Using equilibria relations, we obtain:

$$
\begin{aligned}
\dot{V}= & \left(N_{S i}-N_{S i}^{*}\right)\left(c_{33} N_{S i}^{*}+c_{36} S_{r c}^{*}-c_{33} N_{S i}-c_{36} S_{r c}\right)+d\left(S_{r c}-S_{r c}^{*}\right)\left(c_{64} N_{S i}^{*}\right. \\
& \left.+c_{66} S_{r c}^{*}-c_{64} N_{S i}-c_{66} S_{r c}\right) \\
= & -c_{33}\left(N_{S i}-N_{S i}^{*}\right)^{2}-d c_{66}\left(S_{r c}-S_{r c}^{*}\right)^{2}-c_{36}\left(N_{S i}-N_{S i}^{*}\right)\left(S_{r c}-S_{r c}^{*}\right) \\
& -d c_{64}\left(N_{S i}-N_{S i}^{*}\right)\left(S_{r c}-S_{r c}^{*}\right) \\
= & -c_{33}\left(N_{S i}-N_{S i}^{*}\right)^{2}-d c_{66}\left(S_{r c}-S_{r c}^{*}\right)^{2}-\left(c_{36}+d c_{64}\right)\left(N_{S i}-N_{S i}^{*}\right)\left(S_{r c}-S_{r c}^{*}\right) .
\end{aligned}
$$

We choose $d=\frac{c_{66} a_{3}^{2}}{c_{33} a_{6}^{2}}$. With this and using (8) we can show

$$
\left(c_{36}+d c_{64}\right)^{2}-4 d c_{33} c_{66}<0,
$$

then $\dot{V}$ is definite negative and hence the equilibrium $\left(N_{S i}^{*}, S_{r c}^{*}, N_{M}^{*}\right)$ is GAS. Then, under the condition (8), $\left(N_{S i}^{*}, S_{r c}^{*}, N_{M}^{*}\right)$ is GAS.

Remark 2.1. It is also possible to prove the GAS of $\left(N_{S i}^{*}, S_{r c}^{*}\right)$ by using Dulac criterion [25] with the function $\rho\left(N_{S i}, S_{r c}\right)=\frac{1}{N_{S i} S_{r c}}$ defined on the set $\mathcal{U}=] 0, \frac{b_{3}-d_{3}}{c_{33}}[\times] 0, \frac{b_{6}-d_{6}}{c_{66}}[$.

We have $\frac{\partial\left(\rho X_{1}\right)}{\partial N_{S i}}+\frac{\partial\left(\rho X_{2}\right)}{\partial S_{r c}}=-\left(\frac{c_{33}}{S_{r c}}+\frac{c_{66}}{N_{S i}}\right)<0$. 
Therefore, under the assumption (8) and using Theorem 2.1, the stability properties of system (10) on the set $\mathcal{D}$ are the same as those of the following reduced system

$$
\left\{\begin{aligned}
\frac{d H_{i}}{d t} & =t_{15}\left(N_{H}-H_{i}\right) N_{S i}^{*} s_{i}-r_{12} H_{i} \\
\frac{d s_{e}}{d t} & =\left(t_{32} H_{i}+t_{38} N_{M}^{*} m_{i}\right)\left(1-s_{e}-s_{i}\right)-\left(b_{3}+r_{54}\right) s_{e} \\
\frac{d s_{i}}{d t} & =r_{54} s_{e}-b_{3} s_{i} \\
\frac{d m_{i}}{d t} & =t_{75} N_{S i}^{*} s_{i}\left(1-m_{i}\right)-b_{7} m_{i}
\end{aligned}\right.
$$

defined on the set

$$
\mathcal{D}_{1}=\left\{0 \leq H_{i} \leq N_{H}, 0 \leq s_{e}+s_{i} \leq 1,0 \leq m_{i} \leq 1\right\}
$$

\section{The Disease-free equilibrium and the basic reproductive number}

In this section, we will give an analytic expression for $\mathcal{R}_{0}$ the basic reproductive number of the system (for more details concerning the definition and the computation of $\mathcal{R}_{0}$ one can see $[20,9,5]$ ), and completely answer the stability question for the disease-free equilibrium (DFE). As usual $\rho(M)$ is the spectral radius of the matrix $M$.

\section{1. $\mathcal{R}_{0}$ and the local stability of the DFE}

Proposition 3.1. The DFE for system (10) is the origin $\left(H_{i}, s_{e}, s_{i}, m_{i}\right)=$ $(0,0,0,0)=E_{0}$ and the basic reproduction ratio is given by:

$$
\mathcal{R}_{0}=\sqrt{\frac{r_{54} N_{S i}^{*}\left(b_{7} t_{15} t_{32} N_{H}+r_{12} t_{38} t_{75} N_{M}^{*}\right)}{b_{3} b_{7} r_{12}\left(b_{3}+r_{54}\right)}}=T_{0}^{1 / 2}
$$

Moreover The DFE is LAS if $T_{0}<1$ and is unstable if $T_{0}>1$.

Proof. It is clear that the DFE is $E_{0}=(0,0,0,0)$. Using, the now standard 
techniques $[9,20]$, it is easy to show that the basic reproduction ratio is

$$
\left.\mathcal{R}_{0}=\rho\left(\begin{array}{cccc}
0 & \frac{r_{54} t_{15} N_{H} N_{S i}^{*}}{b_{3}^{2}+r_{54} b_{3}} & \frac{t_{15} N_{H} N_{S i}^{*}}{b_{3}} & 0 \\
\frac{t_{32}}{r_{12}} & 0 & 0 & \frac{t_{38} N_{M}^{*}}{b_{7}} \\
0 & 0 & 0 & 0 \\
0 & \frac{r_{54} t_{75} N_{S i}^{*}}{b_{3}^{2}+r_{54} b_{3}} & \frac{t_{75} N_{S i}^{*}}{b_{3}} & 0
\end{array}\right)\right) .
$$

A simple computation gives:

$$
\mathcal{R}_{0}=\sqrt{\frac{r_{54} N_{S i}^{*}\left(b_{7} t_{15} t_{32} N_{H}+r_{12} t_{38} t_{75} N_{M}^{*}\right)}{b_{3} b_{7} r_{12}\left(b_{3}+r_{54}\right)}}=T_{0}^{1 / 2} .
$$

The Jacobian matrix of (3) at $E_{0}$ is

$$
J_{0}=\left(\begin{array}{cccc}
-r_{12} & 0 & t_{15} N_{H} N_{S i}^{*} & 0 \\
t_{32} & -\left(b_{3}+r_{54}\right) & 0 & t_{38} N_{M}^{*} \\
0 & r_{54} & -b_{3} & 0 \\
0 & 0 & t_{75} N_{S i}^{*} & -b_{7}
\end{array}\right)
$$

$J_{0}$ is a Metzler matrix and we can write $J_{0}=F+V$ with

$$
F=\left(\begin{array}{cccc}
0 & 0 & t_{15} N_{H} N_{S i}^{*} & 0 \\
t_{32} & 0 & 0 & t_{38} N_{M}^{*} \\
0 & 0 & 0 & 0 \\
0 & 0 & t_{75} N_{S i}^{*} & 0
\end{array}\right), V=\left(\begin{array}{cccc}
-r_{12} & 0 & 0 & 0 \\
0 & -\left(b_{3}+r_{54}\right) & 0 & 0 \\
0 & r_{54} & -b_{3} & 0 \\
0 & 0 & 0 & -b_{7}
\end{array}\right) .
$$

We have $F>0$ and $V$ is Metzler stable, see [3, 12, 10,4]. Thanks to Varga's Theorem in [21]: $s\left(J_{0}\right) \leq 0$ iff $\rho\left(-F V^{-1}\right) \leq 1$. Since $\rho\left(-F V^{-1}\right)=\mathcal{R}_{0}$, we then deduce that $E_{0}$ is LAS if $\mathcal{R}_{0}<1$ and is unstable if $\mathcal{R}_{0}>1$.

\subsection{Global stability of the DFE}

We have the following global behavior for system (3) when $\mathcal{R}_{0} \leq 1$.

Theorem 3.1. If $\mathcal{R}_{0} \leq 1$ then the DFE is GAS. 
Proof. Consider the candidate Lyapunov function:

$$
V=\frac{t_{32}}{r_{12}} H_{i}+s_{e}+\frac{b_{3}+r_{54}}{r_{54}} s_{i}+\frac{t_{38} N_{M}^{*}}{b_{7}} m_{i} .
$$

Its derivative along the solutions of (10) satisfies:

$$
\begin{aligned}
\dot{V}= & \frac{t_{32}}{r_{12}} t_{15}\left(N_{H}-H_{i}\right) s_{i} N_{S i}^{*}-t_{32} H_{i} \\
& +\left(t_{32} H_{i}+t_{38} N_{M}^{*} m_{i}\right)\left(1-s_{e}-s_{i}\right)-\left(b_{3}+r_{54}\right) s_{e} \\
& +\frac{b_{3}+r_{54}}{r_{54}}\left(r_{54} s_{e}-b_{3} s_{i}\right)+\frac{t_{38} N_{M}^{*}}{b_{7}}\left(t_{75} N_{S i}^{*} s_{i}\left(1-m_{i}\right)-b_{7} m_{i}\right) \\
=\quad & -t_{32} H_{i} s_{i}-t_{38} N_{M}^{*} m_{i} s_{i} \\
& +s_{e}\left(\frac{b_{3}+r_{54}}{r_{54}} r_{54}-\left(b_{3}+r_{54}\right)-t_{32} H_{i}-t_{38} N_{M}^{*} m_{i}\right) \\
& +s_{i}\left(\frac{t_{32}}{r_{12}} t_{15} N_{S i}^{*}\left(N_{H}-H_{i}\right)-\frac{b_{3}+r_{54}}{r_{54}} b_{3}+\frac{t_{38} t_{75} N_{M}^{*}}{b_{7}} N_{S i}^{*}\left(1-m_{i}\right)\right) \\
= & -\left(s_{e}+s_{i}\right)\left(t_{32} H_{i}+t_{38} N_{M}^{*} m_{i}\right) \\
& +s_{i}\left(\frac{t_{32}}{r_{12}} t_{15} N_{S i}^{*}\left(N_{H}-H_{i}\right)-\frac{b_{3}+r_{54}}{r_{54}} b_{3}+\frac{t_{38} t_{75} N_{M}^{*}}{b_{7}} N_{S i}^{*}\left(1-m_{i}\right)\right) .
\end{aligned}
$$

Hence

$$
\begin{aligned}
\dot{V} \leq & -\left(s_{e}+s_{i}\right)\left(t_{32} H_{i}+t_{38} N_{M}^{*} m_{i}\right) \\
& +s_{i}\left(\frac{t_{32}}{r_{12}} t_{15} N_{S i}^{*} N_{H}-\frac{b_{3}+r_{54}}{r_{54}} b_{3}+\frac{t_{38} t_{75} N_{M}^{*}}{b_{7}} N_{S i}^{*}\right) \\
= & -\left(s_{e}+s_{i}\right)\left(t_{32} H_{i}+t_{38} N_{M}^{*} m_{i}\right) \\
& +s_{i} \frac{b_{3}+r_{54}}{r_{54}} b_{3}\left(\frac{r_{54} N_{S i}^{*}\left(b_{7} t_{15} t_{32} N_{H}+r_{12} t_{38} t_{75} N_{M}^{*}\right)}{b_{3}\left(b_{3}+r_{54}\right) b_{7} r_{12}}-1\right) .
\end{aligned}
$$

Therefore $\dot{V} \leq-\left(s_{e}+s_{i}\right)\left(t_{32} H_{i}+t_{38} N_{M}^{*} m_{i}\right)+\frac{b_{3}+r_{54}}{r_{54}} b_{3}\left(\mathcal{R}_{0}^{2}-1\right) s_{i} \leq 0$ if $\mathcal{R}_{0} \leq 1$.

If $\mathcal{R}_{0}<1$, then $\dot{V}=0$ implies $s_{i}=s_{e}=0$, or $s_{i}=m_{i}=H_{i}=0$ and if $\mathcal{R}_{0}=1$, then $\dot{V}=0$ implies $s_{i}=s_{e}=0$, or $m_{i}=H_{i}=0$. However, in each case the largest invariant subset of $\dot{V}=0$ is the singleton $E_{0}$. Thus, by LaSalle Invariance Principle [14], $E_{0}$ is GAS on $\mathcal{D}_{1}$ when $\mathcal{R}_{0} \leq 1$. 


\section{Endemic Equilibrium}

\subsection{Existence and uniqueness of the endemic equilibrium}

We have shown that the disease-free equilibrium is globally asymptotically stable when $\mathcal{R}_{0} \leq 1$. In the following, we will prove that system (10) has a unique endemic equilibrium when $\mathcal{R}_{0}>1$. To this end we formulate the problem as a fixed point problem. Then we use a theorem due to Hethcote and Thieme ([19], Theorem 2.1) for the existence and uniqueness of a positive fixed point of a multi-variable function. To be self contained, we recall hereafter the result of Hethcote and Thieme:

Theorem 4.1. ([19], Theorem 2.1) Let $F(x)$ be a continuous, monotone non-decreasing, strictly sublinear, bounded function which maps the nonnegative orthant $\mathbb{R}_{+}^{n}=[0, \infty)$ into itself. Let $F(0)=0$ and $F^{\prime}(0)$ exists

and be irreducible. Then $F(x)$ does not have a non-trivial fixed point on the boundary of $\mathbb{R}_{+}^{n}$. Moreover, $F(x)$ has a positive fixed point iff $\rho\left(F^{\prime}(0)\right)>1$. If there is a positive fixed point, then it is unique.

An equilibrium point $\left(H_{i}^{*}, s_{e}^{*}, s_{i}^{*}, m_{i}^{*}\right)$ for system (10) satisfies

$$
\begin{gathered}
s_{i}^{*}=\frac{r_{54}}{b_{3}} s_{e}^{*}, \\
t_{15}\left(N_{H}-H_{i}^{*}\right) s_{i}^{*} N_{S i}^{*}-r_{12} H_{i}^{*}=0 .
\end{gathered}
$$

This implies

$$
H_{i}^{*}=\frac{t_{15} N_{S i}^{*} N_{H} \frac{r_{54}}{b_{3}} s_{e}^{*}}{r_{12}+t_{15} N_{S i}^{*} \frac{r_{54}}{b_{3}} s_{e}^{*}} .
$$

Solving the equations in (10) at steady state gives

$$
s_{e}^{*}=\frac{b_{3}}{r_{54}} s_{i}^{*}, \quad m_{i}^{*}=\frac{t_{75} N_{S i}^{*}}{t_{75} N_{S i}^{*} s_{i}^{*}+b_{7}} s_{i}^{*}, \quad H_{i}^{*}=\frac{t_{15} N_{H} N_{S i}^{*} s_{i}^{*}}{t_{15} N_{S i}^{*} s_{i}^{*}+r_{12}} .
$$

Thus

$$
s_{e}^{*}=\frac{\left(t_{32} H_{i}^{*}+t_{38} N_{M}^{*} m_{i}^{*}\right)}{\left(b_{3}+r_{54}\right)+\left(t_{32} H_{i}^{*}+t_{38} N_{M}^{*} m_{i}^{*}\right)\left(1+\frac{r_{54}}{b_{3}}\right)} .
$$


The fourth equation gives

$$
m_{i}^{*}=\frac{N_{S i}^{*} t_{75} \frac{r_{54}}{b_{3}} s_{e}^{*}}{b_{7}+N_{S i}^{*} t_{75} \frac{r_{54}}{b_{3}}} .
$$

We write this as

$$
U=F(U)
$$

where $U=\left(\begin{array}{c}H_{i} \\ s_{e} \\ m_{i}\end{array}\right)$, and

$$
F=\left(\begin{array}{c}
F_{1} \\
F_{2} \\
F_{3}
\end{array}\right)=\left(\begin{array}{c}
\frac{t_{15} N_{S i}^{*} N_{H} \frac{r_{54}}{b_{3}} s_{e}}{r_{12}+t_{15} N_{S i}^{*} \frac{r_{54}}{b_{3}} s_{e}} \\
\frac{\left(t_{32} H_{i}+t_{38} N_{M}^{*} m_{i}\right)}{\left(b_{3}+r_{54}\right)+\left(t_{32} H_{i}+t_{38} N_{M}^{*} m_{i}\right)\left(1+\frac{r_{54}}{b_{3}}\right)} \\
\frac{N_{S i}^{*} t_{75} \frac{r_{54}}{b_{3}} s_{e}}{b_{7}+N_{S i}^{*} t_{75} \frac{r_{54}}{b_{3}} s_{e}}
\end{array}\right) .
$$

It is easy to remark that $F_{1} \leq N_{H}, F_{2} \leq 1$ and $F_{3} \leq 1$.

The equilibrium points satisfy the relation $U=F(U)$. We use this formulation to prove existence and uniqueness of an endemic equilibrium point.

Let $\Omega=\left\{\left(H_{i}, s_{e}, m_{i}\right): 0 \leq H_{i} \leq N_{H}, 0 \leq s_{e} \leq 1,0 \leq m_{i} \leq 1\right\}$. The function $F$ is a continuous bounded function that maps $\Omega$ into itself and it is infinitely differentiable with Jacobian

$$
F^{\prime}(U)=\left(\begin{array}{ccc}
0 & J_{12} & 0 \\
J_{21} & 0 & J_{23} \\
0 & J_{32} & 0
\end{array}\right)
$$

where: 


$$
\begin{aligned}
J_{12} & =\frac{b_{3} r_{12} r_{54} t_{15} N_{H} N_{S i}^{*}}{\left(b_{3} r_{12}+r_{54} t_{15} N_{S i}^{*} s_{e}\right)^{2}}, J_{21}=\frac{b_{3}^{2} t_{32}}{\left(b_{3}+r_{54}\right)\left(b_{3}+t_{32} H_{i}+t_{38} N_{M}^{*} m_{i}\right)^{2}}, \\
J_{23} & =\frac{b_{3}^{2} t_{38} N_{M}^{*}}{\left(b_{3}+r_{54}\right)\left(b_{3}+t_{32} H_{i}+t_{38} N_{M}^{*} m_{i}\right)^{2}}, J_{32}=\frac{b_{3} b_{7} r_{54} t_{75} N_{S i}^{*}}{\left(b_{3} b_{7}+r_{54} t_{75} N_{S i}^{*} s_{e}\right)^{2}} .
\end{aligned}
$$

The off-diagonal elements of the matrix $F^{\prime}(U)$ are non-negative. Thus the function $F(U)$ is monotone non-decreasing. We have $F(0)=0$ and $\rho\left(F^{\prime}(0)\right)=$ $\mathcal{R}_{0}>1$. Thanks to the graph theory, we claim that $F^{\prime}(0)$ is irreducible because the associated graph of the matrix is strongly connected.

Let us now prove that $F$ is strictly sublinear in $\Omega$, i.e., $F(\lambda U)>\lambda F(U)$, for any $U \in \Omega$ with $U>0$, and $\lambda \in(0,1)$. Some calculations give

$$
\begin{gathered}
\frac{\lambda F_{1}(U)}{F_{1}(\lambda U)}=\frac{r_{12}+t_{15} N_{S i}^{*} \frac{r_{54}}{b_{3}} r s_{e}}{r_{12}+t_{15} N_{S i}^{*} \frac{r_{54}}{b_{3}} s_{e}}<1 . \\
\frac{\lambda F_{2}(U)}{F_{2}(\lambda U)}=\frac{\left(b_{3}+r_{54}\right)+\left(t_{32} H_{i}+t_{38} N_{M}^{*} m_{i}\right)\left(1+\frac{r_{54}}{b_{3}}\right)}{\left(b_{3}+r_{54}\right)+r\left(t_{32} H_{i}+t_{38} N_{M}^{*} m_{i}\right)\left(1+\frac{r_{54}}{b_{3}}\right)}<1 . \\
\frac{\lambda F_{3}(U)}{F_{3}(\lambda U)}=\frac{b_{7}+N_{S i}^{*} t_{75} \frac{r_{54}}{b_{3}} \lambda s_{e}}{b_{7}+N_{S i}^{*} t_{75} \frac{r_{54}}{b_{3}} s_{e}}<1 .
\end{gathered}
$$

So the function $F(U)$ is strictly sublinear. In this way we have proved the following theorem

Theorem 4.2. If $\mathcal{R}_{0} \leq 1$, the only equilibrium point of the system is the disease-free equilibrium $E_{0}$. If $\mathcal{R}_{0}>1$, there also exists a unique endemic equilibrium $E^{*}$ in int $(\Omega)$ whose coordinates satisfy (11).

\subsection{Local Stability of the Endemic Equilibrium}

In this section, we shall prove the local stability of the endemic equilibrium when $\mathcal{R}_{0}>1$. For this we shall follow the method given by Hethcote and Thieme, which is based on a Krasnoselskii technique [19]. A usual way to prove the local asymptotic stability of an equilibrium point $\bar{x}$ of the system of differential equations $\dot{x}=f(x)$ is to prove that the linearized equation $\dot{z}=D f(\bar{x}) z$ has no solutions of the form

$$
z(t)=Z \exp (w t)
$$


with $Z \in \mathbb{C}^{n} \backslash\{0\}, w \in \mathbb{C}$ and $\operatorname{Re}(w) \geq 0$, i.e., $w Z=\operatorname{Df}(\bar{x}) Z$ with $Z \in \mathbb{C}^{n} \backslash\{0\}, w \in \mathbb{C}$ implies $\operatorname{Re}(w)<0$.

Substituting a solution of the form (12) in the linearized equation at the endemic equilibrium, we obtain the following linear equations.

$$
\left\{\begin{aligned}
w Z_{1}= & -\left(t_{15} s_{i}^{*} N_{S i}^{*}+r_{12}\right) Z_{1}+t_{15}\left(N_{H}-H_{i}^{*}\right) N_{S i}^{*} Z_{3} \\
w Z_{2}= & \left(1-s_{e}^{*}-s_{i}^{*}\right) t_{32} Z_{1}-\left(t_{32} H_{i}^{*}+t_{38} N_{M}^{*} m_{i}^{*}\right) Z_{2} \\
& -\left(b_{3}+r_{54}\right) Z_{2}-\left(t_{32} H_{i}^{*}+t_{38} N_{M}^{*} m_{i}^{*}\right) Z_{3} \\
& +N_{M}^{*} t_{38}\left(1-s_{e}-s_{i}\right) Z_{4} \\
w Z_{3}= & r_{54} Z_{2}-b_{3} Z_{3}, \\
w Z_{4}= & t_{75} N_{S i}^{*}\left(1-m_{i}^{*}\right) Z_{3}-\left(t_{75} N_{S i}^{*} s_{i}^{*}+b_{7}\right) Z_{4} .
\end{aligned}\right.
$$

Solving for $Z_{3}$ from the third equation of (13), and substituting the result into the second equation (and simplifying), gives the equivalent system

$$
\left\{\begin{array}{l}
\left(1+\frac{w+t_{15} s_{i}^{*} N_{S i}^{*}}{r_{12}}\right) Z_{1}=\frac{t_{15}\left(N_{H}-H_{i}^{*}\right) N_{S i}^{*}}{r_{12}} Z_{3} \\
\left(1+G_{2}(w)\right) Z_{2}=\frac{1-s_{e}^{*}-s_{i}^{*}}{b_{3}+r_{54}}\left(t_{32} Z_{1}+\left(t_{38} N_{M}^{*} m_{i}^{*}\right) Z_{4}\right) \\
\left(1+\frac{w}{b_{3}}\right) Z_{3}=\frac{r_{54}}{b_{3}} Z_{2} \\
\left(1+\frac{t_{75} N_{S i}^{*} s_{i}^{*}}{b_{7}}\right) Z_{4}=\frac{t_{75} N_{S i}^{*}\left(1-m_{i}^{*}\right)}{b_{7}} Z_{3}
\end{array}\right.
$$

where

$$
G_{2}(w)=\frac{w}{b_{3}+r_{54}}+\frac{\left(t_{32} H_{i}+t_{38} N_{M}^{*} m_{i}\right)}{b_{3}+r_{54}}\left(1+\frac{r_{54}}{w+b_{3}}\right) .
$$

Denoting in the same way

$$
G_{1}(w)=\frac{w+t_{15} N_{S i}^{*} s_{i}^{*}}{r_{12}}, G_{3}(w)=\frac{w}{b_{3}}, G_{4}(w)=\frac{w+t_{75} N_{S i}^{*} s_{i}^{*}}{b_{7}}
$$


we obtain the system

$$
\left\{\begin{array}{l}
{\left[1+G_{1}(w)\right] Z_{1}=(H Z)_{3}} \\
{\left[1+G_{2}(w)\right] Z_{2}=(H Z)_{1}+(H Z)_{4}} \\
{\left[1+G_{3}(w)\right] Z_{3}=(H Z)_{2}} \\
{\left[1+G_{4}(w)\right] Z_{4}=(H Z)_{3}}
\end{array}\right.
$$

with

$$
H=\left(\begin{array}{cccc}
0 & 0 & \frac{t_{15}\left(N_{H}-H_{i}^{*}\right) N_{S i}^{*}}{r_{12}} & 0 \\
\frac{t_{32}\left(1-s_{e}^{*}-s_{i}^{*}\right)}{b_{3}+r_{54}} & 0 & 0 & \frac{t_{38} N_{M}^{*}\left(1-s_{e}^{*}-s_{i}^{*}\right)}{b_{3}+r_{54}} \\
0 & \frac{r_{54}}{b_{3}} & 0 & 0 \\
0 & 0 & \frac{t_{75} N_{S i}^{*}\left(1-m_{i}^{*}\right)}{b_{7}} & 0
\end{array}\right) .
$$

System (14) can spells in a compact way as follows

$$
\left(1+G_{i}(w)\right) Z_{i}=(H Z)_{i},
$$

where $Z=\left(Z_{1}, Z_{2}, Z_{3}, Z_{4}\right)$ with the lexicographic order. Note that the notation $(H Z)_{i}$ (with $\left.i=1, \ldots, 4\right)$ ) denotes the $i$ th coordinate of the vector $H Z$. It should further be noted that the matrix $H$ has non-negative entries, and the equilibrium $E^{*}=\left(H_{i}^{*}, s_{e}^{*}, s_{i}^{*}, m_{i}^{*}\right)$ satisfies $E^{*}=H E^{*}$. Furthermore, since the coordinates of $E^{*}$ are all positive, it follows then that if $Z$ is a solution of (14), then it is possible to find a minimal positive real number $\rho$, depending on $Z$, such that

$$
|Z| \leq \rho E^{*}
$$

where $|Z|=\left(\left|Z_{1}\right|,\left|Z_{2}\right|,\left|Z_{3}\right|,\left|Z_{4}\right|\right)$ and $|\cdot|$ is a norm in $\mathbb{C}$. Now we want to show that $\operatorname{Re}(w)<0$. We assume that $\operatorname{Re}(w) \geq 0$. From this assumption one has two cases : $w=0$ and $w \neq 0$. In the first case, the determinant $\Delta$ of the homogeneous linear system (13) in the variable $Z_{i}(i=1, \ldots, 4)$ 
corresponds to the determinant of the matrix

$$
\left(\begin{array}{cccc}
-1-G_{1}(0) & 0 & \frac{t_{15} N_{S i}^{*}\left(N_{H}-H_{i}^{*}\right)}{r_{12}} & 0 \\
\frac{t_{32}\left(1-s_{e}^{*}-s_{i}^{*}\right)}{b_{3}+r_{54}} & -1-G_{2}(0) & 0 & \frac{t_{38} N_{M}^{*}\left(1-s_{e}^{*}-s_{i}^{*}\right)}{b_{3}+r_{54}} \\
0 & \frac{r_{54}}{b_{3}} & -1-G_{3}(0) & 0 \\
0 & 0 & \frac{t_{75} N_{S i}^{*}\left(1-m_{i}^{*}\right)}{b_{7}} & -1-G_{4}(0)
\end{array}\right) .
$$

Tacking into account that $G_{3}(0)=0$, some elementary calculations give

$$
\begin{aligned}
\Delta & =\left(1+G_{4}(0)\right)\left(1+G_{1}(0)+G_{2}(0)+G_{1}(0) G_{2}(0)\right) \\
& -\frac{1-s_{e}^{*}-s_{i}^{*}}{\left(b_{3}+r_{54}\right) s_{e}^{*}}\left(t_{32} H_{i}^{*}\left(1+G_{4}(0)\right)+t_{38} N_{M}^{*} m_{i}^{*}\left(1+G_{1}(0)\right)\right) .
\end{aligned}
$$

Denoting $\alpha=\max \left\{1+G_{1}(0), 1+G_{4}(0)\right\}$, and using the equilibrium relation $\left(t_{32} H_{i}^{*}+t_{38} N_{M}^{*} m_{i}^{*}\right)\left(1-s_{e}^{*}-s_{i}^{*}\right)-\left(b_{3}+r_{54}\right) s_{e}^{*}=0$, we have

$$
\Delta>\left(1+G_{4}(0)\right)\left(1+G_{1}(0)\right)\left(1+G_{2}(0)\right)-\alpha .
$$

This shows that $\Delta>0$ since $G_{1}(0), G_{2}(0)$, and $G_{4}(0)$ are positive. Therefore, for $w=0$, the only solution of the system (14) is the trivial one $Z=0$ which implies that $w \neq 0$. Assume now that $w \neq 0$, and $\mathcal{R} e w \geq 0$. Let $G(w)=\min \left\{\left|1+G_{i}(w)\right|, i=1, \ldots, 4\right\}$. It is easy to prove that in this case $\left|1+G_{i}(w)\right|>1$ for all $i$, and therefore $G(w)>1$. Taking norms on both sides of (14), and using the fact that $H$ is non-negative, we obtain the following inequality:

$$
G(w)|Z| \leq H|Z|
$$

Using (15) and (16), we get

$$
G(w)|Z| \leq \rho H E^{*}=\rho E^{*} .
$$

Which implies

$$
|Z| \leq \frac{\rho}{G(w)} E^{*}<\rho E^{*}
$$

But this contradicts the minimality of $\rho$. Therefore $\operatorname{Re}(w)<0$. We have then proved the following theorem. 
Theorem 4.3. If $\mathcal{R}_{0}>1$, then the positive endemic equilibrium $E^{*}$ of the system (10) is locally asymptotically stable.

\subsection{Global Stability of the Endemic Equilibrium}

In this section we will establish the global stability of the unique endemic equilibrium point when $\mathcal{R}_{0}>1$. We shall use the properties of K-monotone systems for the analysis of our system (see [18]). We begin by recalling the definition of K-monotone systems as well as a result concerning convergence properties of strongly monotone systems. Let

$$
\dot{x}=f(x)
$$

where $f$ is a continuously differentiable function defined on a convex, open set $U$ in $\mathbb{R}^{n}$. Let $q=\left(q_{1}, \ldots, q_{n}\right), q_{i} \in\{0,1\}, 1 \leq i \leq n$, and $K=$ $\left\{x \in \mathbb{R}^{n}:(-1)^{q_{i}} x_{i} \geq 0,1 \leq i \leq n\right\}$. The solution $\phi_{t}(x)$ of (17) preserves the partial ordering $\leq_{K}$ (for $t \geq 0$ ) and system (17) is said to be of type $K$ monotone if whenever $x, y \in U$ with $x \leq_{K} y$ then $\phi_{t}(x) \leq_{K} \phi_{t}(y)$ for all $t \geq 0$ for which both $\phi_{t}(x)$ and $\phi_{t}(y)$ are defined. The following lemma gives necessary and sufficient conditions for (17) to be a type $K$-monotone system in the case that $f \in \mathcal{C}^{1}(U)$ for an open convex set $U \in \mathbb{R}^{n}$.

Lemma 4.1. (Smith [18], Lemma 2.1) If $f \in \mathcal{C}^{1}(U)$ where $U$ is open and convex in $\mathbb{R}^{n}$ then $\phi_{t}$ preserves the partial ordering $\leq_{K}$ for $t \geq 0$ if only if $P_{q} \operatorname{Df}(x) P_{q}$ has nonnegative off-diagonal elements for every $x \in U$, where $P_{q}=\operatorname{diag}\left((-1)^{q_{1}}, \ldots,(-1)^{q_{n}}\right)$.

For strongly monotone systems, we recall the following useful result:

Theorem 4.4. (Hirsh [11], theorem 10.3) Suppose $X$ is an open subset of a strongly ordered topological vector space, and $f$ generates a strongly monotone flow in $X$. Let $W \subset X$ be an open set of points with compact orbit closures, and assume that there is a unique equilibrium $p$ in $\bigcup_{x \in W} \omega(x)$. Then $\phi_{t}(x) \longrightarrow p$ for all $x \in W$.

We shall use this global convergence result to prove the following result

Theorem 4.5. If $\mathcal{R}_{0}>1$, then the positive endemic equilibrium state $E^{*}$ of the system (10) is globally asymptotically stable in the interior of the set $\mathcal{D}_{1}$. 
Proof. We rewrite the system (10) using the variables $\left(H_{i}, s_{s}, s_{i}, m_{i}\right)$ instead of $\left(H_{i}, s_{e}, s_{i}, m_{i}\right)$ used in the previous sections. We get the following system:

$$
\left\{\begin{aligned}
\frac{d H_{i}}{d t} & =t_{15}\left(N_{H}-H_{i}\right) N_{S i}^{*} s_{i}-r_{12} H_{i}, \\
\frac{d s_{s}}{d t} & =b_{3}-\left(t_{32} H_{i}+t_{38} N_{M}^{*} m_{i}+b_{3}\right) s_{s}, \\
\frac{d s_{i}}{d t} & =r_{54}\left(1-s_{s}-s_{i}\right)-b_{3} s_{i}, \\
\frac{d m_{i}}{d t} & =t_{75} N_{S i}^{*} s_{i}\left(1-m_{i}\right)-b_{7} m_{i}
\end{aligned}\right\}=f\left(H_{i}, s_{s}, s_{i}, m_{i}\right)=f(x) .
$$

With Jacobian

$$
\begin{gathered}
D f(x)= \\
\left(\begin{array}{cccc}
-r_{12}-t_{15} N_{S i}^{*} s_{i} & 0 & t_{15} N_{S i}^{*}\left(N_{H}-H_{i}\right) & 0 \\
-t_{32} s_{s} & -b_{3}-t_{32} H_{i}-t_{38} N_{M}^{*} m_{i} & 0 & -t_{38} N_{M}^{*} s_{s} \\
0 & -r_{54} & -b_{3}-r_{54} & 0 \\
0 & 0 & t_{75} N_{S i}^{*}\left(1-m_{i}\right) & -b_{7}-t_{75} N_{S i}^{*} s_{i}
\end{array}\right) .
\end{gathered}
$$

If we choose the matrix $P$ as

$$
P=\left(\begin{array}{cccc}
1 & 0 & 0 & 0 \\
0 & -1 & 0 & 0 \\
0 & 0 & 1 & 0 \\
0 & 0 & 0 & 1
\end{array}\right)
$$

Then the matrix $P \operatorname{Df}(x) P$ is a Metzler matrix, i.e., it has nonnegative offdiagonal elements for all $x \in \mathcal{D}_{1}$. Thus, system (18) is K-monotone in $\stackrel{\mathcal{D}}{1}_{1}$ (the interior of $\mathcal{D}_{1}$ ) with respect to the partial ordering defined by the orthant $K=\left\{H_{i} \geq 0, s_{s} \leq 0, s_{i} \geq 0, m_{i} \geq 0\right\}$. Moreover it is strongly monotone with respect to the orthant $K$ because $D f(x)$ is irreducible.

Thanks Hirsch's theorem 4.4 and the fact that we have only one endemic equilibrium $E^{*}$ in $\mathcal{D}_{1}$ which is locally asymptotically stable when $\mathcal{R}_{0}>1$ we can conclude that $E^{*}$ is globally asymptotically stable in $\mathcal{D}_{1}$ when $\mathcal{R}_{0}>$ 1.

\section{Biological control}

The first way to eradicate the disease is to use a competitor resistant snail species that is able to eliminate the population of the intermediate host 
snails. This means that system (4) admits $E_{2}=\left(0, \frac{a_{6}}{c_{66}}\right)$ as a globally asymptotically stable equilibrium state. This equilibrium corresponds to the absence of the intermediate host snails: $N_{S i}=0$. According to the computations done in Paragraph 2.1, the parameters must satisfy $c_{66} a_{3}-$ $c_{36} a_{6}<0$ and the other equilibrium $E_{1}=\left(\frac{a_{3}}{c_{33}}, 0\right)$ must be unstable which implies that we must have $c_{33} a_{6}-c_{64} a_{3}>0$. Remark that when the two above conditions are satisfied then the coexistence equilibrium $E^{*}$ does not exist.

To summarize, the population of the intermediate host snails can be eliminated if the parameters satisfy the following condition

$$
\frac{a_{6}}{a_{3}}=\frac{b_{6}-d_{6}}{b_{3}-d_{3}}>\max \left\{\frac{c_{66}}{c_{36}}, \frac{c_{64}}{c_{33}}\right\}
$$

This situation is illustrated in Figure 1. The simulation has been done using parameter values from [1].

However the above condition (19) is strong and may be hard to satisfy in practice. Therefore a second method to eradicate the disease without completely eliminating the intermediate host snails is to choose a competitor resistant snail species in such a way that $\mathcal{R}_{0} \leq 1$. Recall that

$$
\mathcal{R}_{0}^{2}=\frac{r_{54} N_{S i}^{*}\left(b_{7} t_{15} t_{32} N_{H}+r_{12} t_{38} t_{75} N_{M}^{*}\right)}{b_{3} b_{7} r_{12}\left(b_{3}+r_{54}\right)} .
$$

In the expression of $\mathcal{R}_{0}$, the only quantity that depends on the competitor resistant snail species is $N_{S i}^{*}$ since (by relation (5)) $N_{S i}^{*}=\frac{c_{36} a_{6}-c_{66} a_{3}}{c_{36} c_{64}-c_{33} c_{66}}$ and the other terms do not depend on the competitor.

We can remark that the introduction of a competitor reduces the value of $\mathcal{R}_{0}$ since the value of the steady state size of the intermediate host snail in the absence of the competitor (which is equal to $\frac{a_{3}}{c_{33}}$ ) is larger than its value in the presence of the competitor.

Now, $\mathcal{R}_{0} \leq 1$ iff

$$
\frac{c_{36} a_{6}-c_{66} a_{3}}{c_{36} c_{64}-c_{33} c_{66}}=N_{S i}^{*} \leq \frac{b_{3} b_{7} r_{12}\left(b_{3}+r_{54}\right)}{r_{54}\left(b_{7} t_{15} t_{32} N_{H}+r_{12} t_{38} t_{75} N_{M}^{*}\right)}
$$


The right-hand side expression of the above inequality (20) depends only on the disease characteristics as well as on the human, mammal and intermediate snail demographic parameters. It does not depend on the resistant competitor snail species.

The competitor characteristics have then to satisfy the inequality (20) in order to eradicate the disease. This means that the resistant competitor snail species has to be able to reduce the steady state size of the intermediate snail species under some quantity that depends on the disease characteristics $\left(r_{12}, r_{54}, t_{15}, t_{32}, t_{75}\right)$, on the demographic parameters of the intermediate snail, and on the human and mammal populations.

\section{Summary and conclusions}

In this paper, we have presented a stability analysis of a deterministic model for the transmission dynamics of a schistosomiasis infection. Eight sub population sizes were considered: human host susceptible and infected, snail intermediate host susceptible, latent, and shedding, resistant competitor snail, mammal host susceptible and infected. The snails competition is used to control the transmission of the disease.

Mathematical properties of the model are analyzed and used to reduce the dimension of the system under consideration.

The reproductive number $\mathcal{R}_{0}$ is then analytically and explicitly computed. We proved that the disease-free steady state $E_{0}$ is globally asymptotically stable if $\mathcal{R}_{0} \leq 1$.

We have also established the existence and uniqueness of an endemic equilibrium $E^{*}$ in the case where $\mathcal{R}_{0}>1$. Using some properties of monotone systems we have proved the global asymptotic stability of the endemic equilibrium when it exists i.e., when $\mathcal{R}_{0}>1$.

In a more realistic situation, the speed of a river should affect the transmission dynamics of schistosomiasis. We are working on this perspective. A model with spatial structure involving the present ODE system coupled with a shallow water equations is being studied, see [8]. 


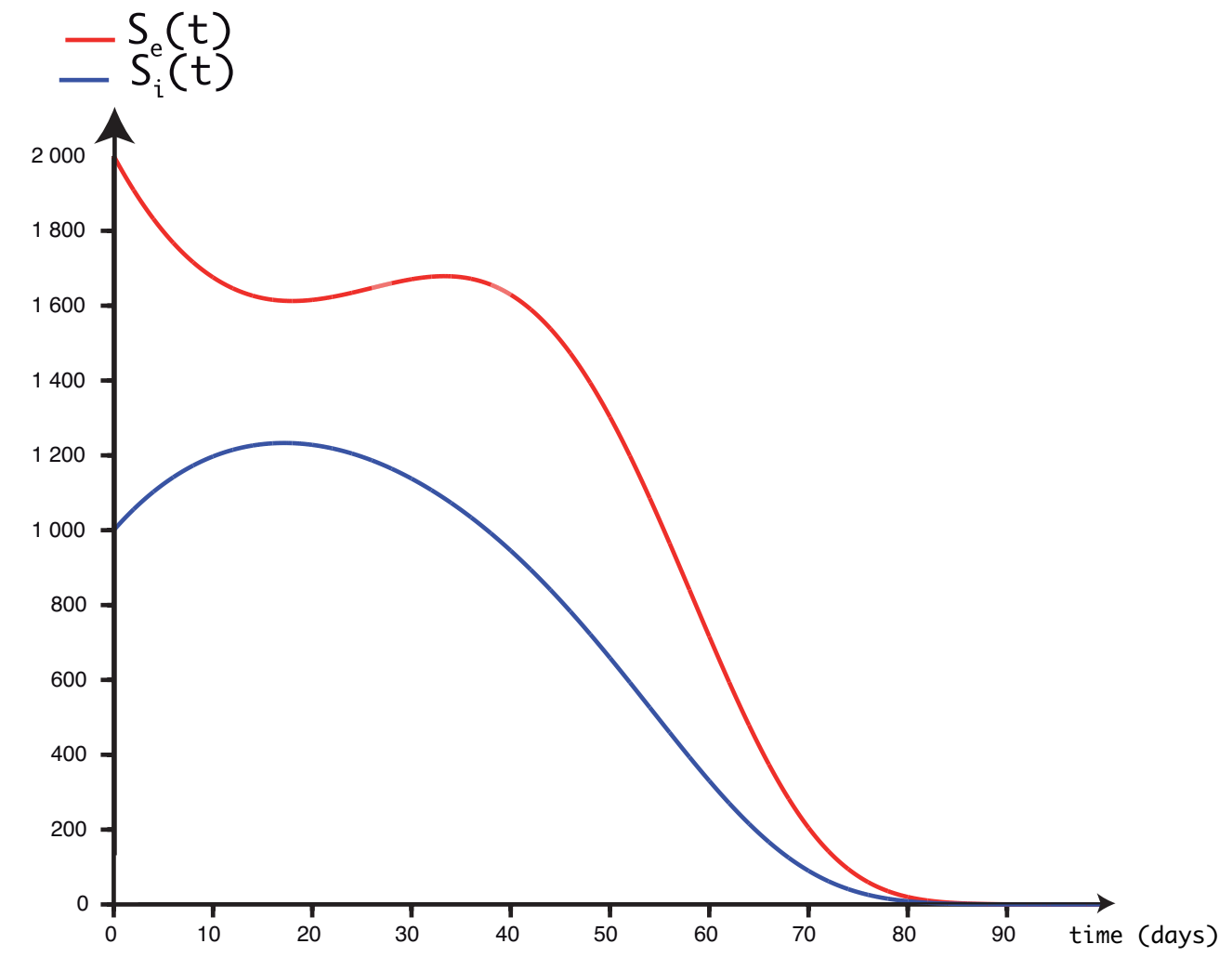

Figure 1: Evolution of the latent $S_{e}(t)$ and infected $S_{i}(t)$ snails when the elimination condition (19) is satisfied.

[1] E. J. Allen and H. D. J. Victory. Modelling and simulation of a schistosomiasis infection with biological control. Acta Trop, 87(2):251-267, 2003.

[2] R. Anderson and R. May. Helminth infections of humans: mathematical models, population dynamics, and control. Advances in parasitology, 24:1-101, 1985.

[3] A. Berman and R. J. Plemmons. Nonnegative matrices in the mathematical sciences. SIAM, 1994.

[4] F. Brauer and C. Castillo-Chávez. Mathematical models in population 
biology and epidemiology, volume 40 of Texts in Applied Mathematics. Springer-Verlag, New York, 2001.

[5] C. Castillo-Chavez, Z. Feng, and W. Huang. On the computation of $R_{0}$ and its role on global stability. In Mathematical approaches for emerging and reemerging infectious diseases: an introduction (Minneapolis, MN, 1999), volume 125 of IMA Vol. Math. Appl., pages 229-250. Springer, New York, 2002.

[6] Z. Chen, L. Zou, D. Shen, W. Zhang, and S. Ruan. Mathematical modelling and control of schistosomiasis in hubei province, china. Acta Tropica, 115(1-2):119-125, 2010.

[7] J. E. Cohen. Mathematical models of schistosomiasis. Annual Review of Ecology and Systematics, 8(1):209-233, 1977.

[8] M. Diaby, A. Iggidr, A. Sene, and M. Sy. Analysis of a schistosomiasis infection model with spatial structure, in progress.

[9] O. Diekmann, J. Heesterbeek, and J. Metz. On the definition and the computation of the basic reproduction ratio $R_{0}$ in models for infectious diseases in heterogeneous populations. J. Math. Biol., 28(4):365-382, 1990 .

[10] O. Diekmann and J. Heesterbeek. Mathematical epidemiology of infectious diseases. Model building, analysis and interpretation. Wiley Series in Mathematical and Computational Biology. Chichester: Wiley. 300 p. , 1999 .

[11] M. W. Hirsch. Stability and convergence in strongly monotone dynamical systems. J. Reine Angew. Math., 383:1-53, 1988.

[12] J. A. Jacquez and C. P. Simon. Qualitative theory of compartmental systems. SIAM Rev., 35(1):43-79, 1993.

[13] A. R. Kimbir. A mathematical model of the transmission dynamics of schistosomiasis. J. Nigerian Math. Soc., 16/17:39-63, 1997/98.

[14] J. LaSalle. The stability of dynamical systems. CBMS-NSF Regional Conference Series in Applied Mathematics. 25. Philadelphia, Pa.: SIAM, Society for Industrial and Applied Mathematics., 1976. 
[15] G. Macdonald. The dynamics of helminth infections, with special reference to schistosomes. Transactions of the Royal Society of Tropical Medicine and Hygiene, 59(5):489-506, 1965.

[16] F. A. Milner and R. Zhao. A deterministic model of schistosomiasis with spatial structure. Math. Biosci. Eng., 5(3):505-522, 2008.

[17] J. P. Pointier and J. Jourdane. Biological control of the snail hosts of schistosomiasis in areas of low transmission: the example of the caribbean area. Acta Tropica, 77(1):53-60, 2000.

[18] H. L. Smith. Systems of Ordinary Differential Equations Which Generate an Order Preserving Flow. A Survey of Results. SIAM Review, 30(1):87-113, 1988.

[19] H. W. Hethcote and H. R. Thieme. Stability of the endemic equilibrium in epidemic models with subpopulations. Math. Biosci., 75:205-227, 1985.

[20] P. van den Driessche and J. Watmough. Reproduction numbers and sub-threshold endemic equilibria for compartmental models of disease transmission. Math. Biosci., 180:29-48, 2002. John A. Jacquez memorial volume.

[21] R. Varga. Matrix iterative analysis. 2. printing. (Prentice-Hall Series in Automatic Computation). Englewood Cliffs, New Jersey:Prentice-Hall, Inc. XIII, 322 p. , 1962.

[22] M. Vidyasagar. Decomposition techniques for large-scale systems with nonadditive interactions: Stability and stabilizability. IEEE Trans. Autom. Control, 25:773-779, 1980.

[23] WHO, Schistosomiasis.

http://www.who.int/mediacentre/factsheets/fs115/en/index.html (2010).

[24] M. E. J. Woolhouse. On the application of mathematical models of schistosome transmission dynamics. i. natural transmission. Acta Tropica, 49(4):241-270, 1991.

[25] J. K. Hale. Ordinary differential equations. Pure and Applied Mathematics, John Wiley $\mathcal{E}$ Sons, New York, 1969. 